\title{
Predicting the Shape of Pointy-Rock Forests
}

\section{The shape and curvature evolution of dissolving rocks can be predicted using a new theory.}

\section{By Rachel Berkowitz}

$\mathrm{O}$ ver long time periods, a water-submerged limestone rock will dissolve to form a structure that contains a series of sharp pinnacles. Researchers think that this structure arises from erosion of the rock by the persistent flows that are generated along the surface of the rock by dense, solute-laden fluid. Models that describe pinnacle growth in this way rely on numerical effects that are not always based in physical reality. Now, Jinzi Mac Huang of New York University Shanghai and Nicholas Moore of the US Naval Academy, Maryland, derive exact solutions to a model that predicts the shape and curvature evolution of these features [1]. The duo says that their finding could aid in understanding the origins of other geological features, such as icicles or stalagmites.

In experiments, Huang and Moore recently demonstrated how a block of solidified sugar placed in a fluid can spontaneously develop a collection of pinnacle structures that resembles those seen in naturally occurring stone forests. They then built a model to describe how the curvature of each pinnacle's tip changes over time. The model accounted for how the

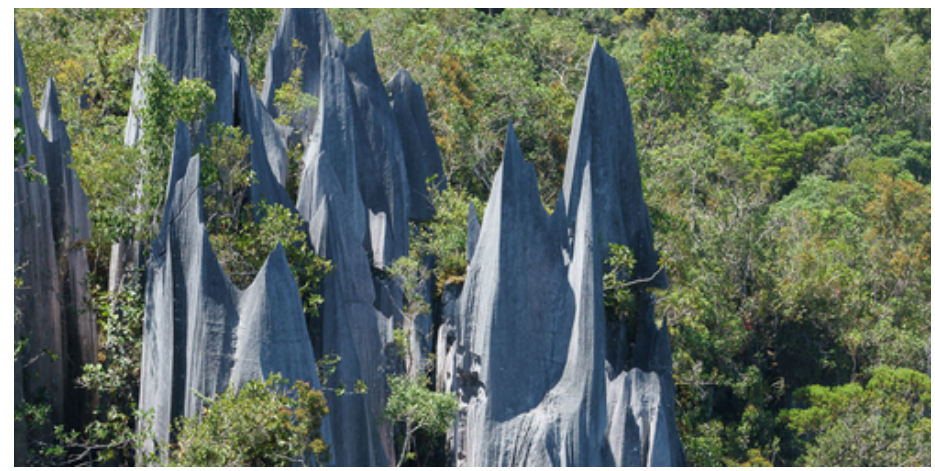

Credit: Grant Dixon Photography dissolution of a rock can induce natural convective flows over its surface that can locally change the rock's dissolution rate. They found that this feedback pushes the pinnacles to become pointier, while a thermodynamic term halts that unbounded growth, leading to more rounded tips.

Now, Huang and Moore derive exact solutions to their model. Their solutions show that the pinnacles should not become infinitely sharp even without the thermodynamic term. Rather, their theory suggests that sharpening slows over time, with the pinnacle geometry converging to a terminal shape that is sharp, slender, and has a finite curvature. The model also identifies a relationship between a rock's initial and final morphology. The model could be used to estimate a geological structure's age and the environmental conditions it has been subjected to.

Rachel Berkowitz is a Corresponding Editor for Physics based in Vancouver, Canada.

\section{REFERENCES}

1. J. M. Huang and N. J. Moore, "Morphological attractors in natural convective dissolution,” Phys. Rev. Lett. 128, 024501 (2022). 